\title{
Molecular epidemiology of meticillin-resistant Staphylococcus aureus (MRSA): think regionally but use globally uniform typing languages
}

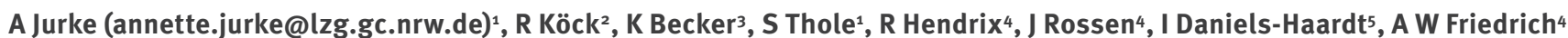

1. Department of Infectiology and Hygiene, NRW Centre for Health, Münster, Germany

2. Institute of Hygiene, University Hospital Münster, Germany

3. Institute of Medical Microbiology, University Hospital Münster, Germany

4. Department of Medical Microbiology, University of Groningen, University Medical Center Groningen, the Netherlands

5. Department of Health Protection, Health Monitoring, NRW Centre for Health, Münster, Germany

To the editor: We appreciate the report by Lekkerkerk et al. 'Seafarers: a new risk group for meticillin-resistant Staphylococcus aureus (MRSA)' on MRSA of unknown origin, detected in seafarers, which indeed seem to play an important role in explaining the diversity of MRSA spa types in an country with low MRSA prevalence as the Netherlands [1].

The report makes clear that the population being at risk can vary from region to region.

As the report describes, it is essential that aside from the commonly known risk factors (e.g. acute care hospital stay in endemic countries), the MRSA-risk population needs to be evaluated on a regional scale and screening protocols need to be adapted according to the result of these evaluations on a regular basis. The goal is to find as many carriers as possible by screening as few patients as necessary. National screening protocols should encourage regional healthcare clusters [2] to assess whether their own risk population is congruent to the one established for the national level.

While seafarers seem to be a major population at risk in harbour cities, farmers are a far more relevant risk group for the Dutch-German border region as demonstrated by the fact that $17 \%$ to $29 \%$ of all MRSA patients detected at hospital admission on the German side of the border in the period from 2006 to 2012 carried livestock-associated (LA) MRSA spa types (predominantly spa CC011, MLST CC398) [3]. In comparison, from 2010 to 2011 only $5.4 \%$ of MRSA patients included in a nationwide German study could be attributed to LA-MRSA (predominantly spa CC011, MLST CC398) [4]. The particular increasing admission prevalence of LA-MRSA in the Dutch-German border region can be explained by the intensive pig farming in this area. Livestock density is known to be associated with occurrence of LA-MRSA in humans [5]. Nearly all studies use common molecular typing methods to distinguish livestock-related isolates from other MRSA. As epidemiology of MRSA seems different from region to region and is changing rapidly, the understanding of the fluctuating epidemiology would not be possible without proper typing.

A retrospective case-control study in the German part of the Dutch-German EUREGIO showed that patients carrying LA-MRSA are different from patients carrying classical hospital-acquired (HA) MRSA with respect to underlying disease, invasive treatment and length of stay in intensive care unit [6]. The key findings of the study were only achievable by molecular spa typing data allowing retrospective stratification of patients into carriers of HA-MRSA versus LA-MRSA. Furthermore, a significant proportion (\$30\%) of patients colonised with LA-MRSA at admission were not associated with contact to farm animals indicating other ways of transmission.

While dissemination of LA-MRSA is discussed here, the report on seafarers makes also clear that without proper molecular typing methods using a globally understandable nomenclature, the unusual MRSA spa types found in seafarers would not have been identified. It also shows that publications on microorganisms that are important to clinical microbiology, infection prevention and public health (as e.g. MRSA and carbapenemase-producing Enterobacteriaceae) benefit from common typing languages based on publicly available reference databases that ensure excellent quality and a unique nomenclature. This is of particular interest in a time when modern technology such as whole genome-based typing is being implemented for clinical microbiology, infection prevention and public health.

Such common typing languages allow the performance of studies that permit to adapt infection control 
measures to the regional need, but from which the results can be also shared and understood worldwide. Moreover, these typing languages own the capacity to become professional tools for daily use in infectious diseases prevention and management.

\section{Conflict of interest}

None declared.

Authors' contributions

Conceived and writing the paper: A Jurke, AW Friedrich; Contributed to writing and discussion: R Köck, K Becker; Contributed to discussion: S Thole, R Hendrix, J Rossen, I Daniels-Haardt.

\section{References}

1. Lekkerkerk WS, van Genderen PJ, Severin JA, Peper JP, Storm EF, Vos MC. Letter to the editor: Seafarers: a new risk group for meticillin-resistant Staphylococcus aureus (MRSA). Euro Surveill. 2013;18(43):pii=20618. Available from: http://www. eurosurveillance.org/ViewArticle.aspx?Articleld $=20618$

2. Ciccolini M, Donker T, Köck R, Mielke M, Hendrix R, Jurke A, et al. Infection prevention in a connected world: The case for a regional approach. Int J Med Microbiol. 2013;303(6-7):380-7. http://dx.doi.org/10.1016/j.ijmm.2013.02.003. PMid:23499307.

3. Köck R, Schaumburg F, Mellmann A, Koksal M, Jurke A Becker K, et al. Livestock-associated methicillin-resistant Staphylococcus aureus (MRSA) as causes of human infection and colonization in Germany. PLoS One. 2013;8(2):e55040. http://dx.doi.org/10.1371/journal.pone.0055040. PMid:23418434. PMCid:PMC3572123.

4. Schaumburg F, Köck R, Mellmann A, Richter L, Hasenberg F, Kriegeskorte A, et al. Population Dynamics among MethicillinResistant Staphylococcus aureus Isolates in Germany during a 6-Year Period. J Clin Microbiol. 2012;50(10):3186-92. http://dx.doi.org/10.1128/JCM.01174-12. PMid:22814464. PMCid:PMC3457438.

5. van Loo I, Huijsdens X, Tiemersma E, de Neeling A, van de Sande-Bruinsma N, Beaujean D et al. Emergence of methicillin-resistant Staphylococcus aureus of animal origin in humans. Emerg Infect Dis. 2007;13(12):1834-9. http://dx.doi.org/10.3201/eid1312.070384. PMid:18258032. PMCid:PMC 2876750.

6. Köck R, Harlizius J, Bressan N, Laerberg R, Wieler LH, Witte W, et al. Prevalence and molecular characteristics of methicillinresistant Staphylococcus aureus (MRSA) among pigs on German farms and import of livestock-related MRSA into hospitals. Eur J Clin Microbiol Infect Dis. 2009;28(11):1375-82. http://dx.doi.org/10.1007/s10096-009-0795-4. PMid:19701815. PMCid:PMC 2772956. 